\title{
Impacto de la dieta sobre la inducción de infección con quistes de Giardia lamblia en ratas Sprague-Dawley
}

\author{
Martha Elvia Díaz-Cinco, M en $C_{1}{ }^{(1)}$ Martha $N$ ydia Ballesteros-Vázquez, $M$ en $C,{ }^{(1)}$ \\ Rosalba Pérez-Morales, Q B, ${ }^{(1)}$ Verónica Mata-Haro, Q B. ${ }^{(1)}$
}

\author{
Díaz-Cinco ME, Ballesteros-Vázquez MN, \\ Pérez-Morales R, Mata-HaroV. \\ Impacto de la dieta sobre la inducción \\ de infección con quistes de Giardia lamblia \\ en ratas Sprague-Dawley \\ Salud Publica Mex 2002;44:315-322.
} El texto completo en inglés de este artículo está disponible en: http://www.insp.mx/salud/index.html

\section{Resumen}

O bjetivo. Evaluar el efecto de la desnutrición en la inducción de la giardiosis con diferentes concentraciones de quistes de Giardia lamblia utilizando como modelo animal ratas Sprague-Dawley. Material y métodos. Es un estudio experimental efectuado entre 1995 y 1999, en el Centro de Investigación en Alimentación y D esarrollo, de la ciudad de Hermosillo, estado de Sonora, México, y cuya muestra estuvo integrada por dos grupos de 6 a 8 unidades experimentales, las cuales recibieron dos tipos de dieta y cinco tratamientos con diferente concentración de inóculo de quistes de Giardia lamblia. Durante la fase experimental se monitoreó la excreción de quistes, ganancia de peso, consumo de alimento, análisis del contenido intestinal y daños macro y microscópicos en mucosa intestinal. El tratamiento estadístico de los datos consistió en análisis de varianza, y de residuales cuando fue necesario. Resultados. En los animales alimentados con una dieta que cubre sus requerimientos nutricionales (DN) se observó que es posible provocar la infección con 60 quistes, mientras que en los animales desnutridos (dieta D LN) la concentración que se requiere es de únicamente seis quistes para establecer daños a nivel de mucosa intestinal. Conclusiones. La ganancia de peso monitoreada durante los 10 días de ensayo no resultó ser un buen indicador de la infección por Giardia lamblia. El porcentaje de infección depende de la concentración de quistes inoculados y del estado de nutrición de

\author{
Díaz-Cinco ME, Ballesteros-Vázquez MN, \\ Pérez-Morales R, Mata-HaroV. \\ The effect of malnourishment \\ on the development of giardiosis \\ in Sprague-Dawley rats. \\ Salud Publica Mex 2002;44:315-322. \\ The English version of this paper \\ is available at: http://www.insp. mx/salud/index.html
}

\begin{abstract}
A bstract
0 bjective.To assess the effect of malnutrition on the development of giardiosis in Sprague-D awley rats, using different inoculum sizes of $\mathrm{G}$ iardia lamblia cysts. Material and Methods. An experimental study was conducted between 1995 and 1999 at C entro de Investigación,A limentación y Desarrollo (Center for Research, Food, and Development), in Hermosillo, Sonora, Mexico. The study po pulation consisted of two groups of six to eight experimental units that were fed two different diets and inoculated five different concentrations of $G$ iardia lamblia cysts. D ata were collected on excretion of cysts, weight gain, food intake, bowel contents, and macro and microscopic lesions in the intestinal mucosa. Statistical analysis consisted of analysis of variance and residuals. Results. Animals fed with a diet meeting nutritional requirements required an infecting inoculum of 60 cysts, while malnourished rats required only six cysts to develop mucosal lesions. Conclusions. Weight gain monitored during ten days was not a good indicator of $G$ iardia lamblia infection. Infection depended on cyst inoculum size as well as on the nutritional status of the tested animals. The English version of this paper is available at: http:// www.insp.mx/salud/index.html
\end{abstract}

El presente trabajo fue realizado gracias al apoyo financiero de la Fundación Mexicana para la Salud.

(1) Centro de Investigación en Alimentación y Desarrollo,A.C., Hermosillo, México.

Fecha de recibido: 6 de julio de 2001 - Fecha de aprobado: 11 de febrero de 2002

Solicitud de sobretiros: Martha Elvia Díaz-C inco. Carretera a laVictoria km 0.6, 83000 Hermosillo, Sonora, México. Correo electrónico: mdiaz@ cascabel.ciad.mx 
Ios animales evaluados. El texto completo en inglés de este artículo está disponible en: http://www.insp.mx/salud/ index.html

Palabras clave: giardiosis; trastornos nutricionales; Giardia lamblia; México
Key words: giardiosis; nutrition disorders; Giardia lamblia; Mexico
$\mathrm{E}$ $\mathrm{n}$ algunos países del mundo, sobre todo en aquellos en desarrollo, se considera que la giardiosis es un problema de salud pública. ${ }^{1}$ En México, afecta aproximadamente a 9 millones de personas y se considera que la población infantil es la más vulnerable. ${ }^{2}$ Específicamente en el estado de Sonora, México, diversos estudios han informado de la presencia de infecciones por Giardia, tanto en población urbana como rural. ${ }^{3,4}$ Uno de los vectores evaluados en esta región ha sido el agua para consumo humano, encontrándose concentraciones de cinco quistes $/ \mathrm{ml}$ en la ciudad de Hermosillo y de 17 en la de Obregón. ${ }^{5}$

De acuerdo con algunos autores, se ha señalado que la giardiosis puede interferir con la absorción de algunos nutrimentos llegándose en algunos casos a provocar un deterioro en el estado nutricio. ${ }^{1}$ En este contexto, un estudio realizado en una comunidad del norte de México notificó que la infección con Giardia en niños provocó mala absorción de hierro y vitamina A, contribuyendo a empeorar el estado de anemia. ${ }^{6}$

Algunos estudios realizados en ratones y jerbos utilizados como modelos animales para reproducir la giardiosis, y que fueron alimentados con todos sus requerimientos nutricionales, han demostrado que entre 16 y 100\% de los animales inoculados quedan infectados y que esta infección depende tanto de la edad del animal como de la concentración del inóculo empleado. En este último punto, la mayoría de los informes indican el uso de inóculos en concentraciones de 100 o más quistes. 7,8

De igual manera, la rata ha sido utilizada como modelo animal para reproducir la infección con Giardia lamblia, ya que se considera que la giardiosis provocada en este modelo tiene gran similitud con la manifestada en humanos y ratones. La rata como modelo se considera que proporciona una herramienta útil para la investigación de la patofisiología de la giardiosis. Un estudio realizado en ratas SpragueDawley reportó la susceptibilidad a la infección con Giardia lamblia, al demostrarse la presencia de quistes o detectar antígenos de Giardia lamblia en heces. ${ }^{9}$

Por otro lado, el estado inmunológico del huésped puede influir tanto en la gravedad de las manifestaciones como en el tiempo de evolución, siendo más frecuente la infección en pacientes inmunosuprimidos. En ensayos hechos con animales inmunosuprimidos, utilizando el medicamento ciclofosfamida, se ha observado un implante de la infección, con una excreción de quistes al sexto día, y daño. Sin embargo, las concentraciones empleadas de inóculo han sido muy grandes, superiores a los 5000 quistes. $^{10}$

Considerando que en el ser humano la desnutrición compromete el estado inmunológico, es de esperarse que haya mayor susceptibilidad a una infección, en cuyo caso la situación se agrava aún más, puesto que parásitos como la Giardia impiden la absorción de algunos nutrimentos.

Considerando que la infección por Giardia es un problema de salud, y que su presencia en pacientes inmunocomprometidos puede empeorar su condición nutricional, aunado esto a la necesidad de contar con más información acerca de la dosis infectiva mínima, el objetivo de esta investigación fue evaluar el efecto de la desnutrición en la inducción de la giardiosis con diferentes concentraciones de quistes de Giardia lamblia, utilizando como modelo animal ratas SpragueDawley.

\section{Material y métodos}

Se utilizaron quistes de Giardia lamblia cepa $\mathrm{CH}-3$ patógena, los cuales fueron adquiridos en el laboratorio P.R.L.-DyNAgenics de la Universidad Estatal de Colorado de Estados Unidos de América. La cepa se mantiene en jerbos SPF libres de patógenos específicos y los quistes se purificaron en el mismo laboratorio.

En nuestro laboratorio, los quistes fueron cuantificados utilizando la cámara de Neubauer. ${ }^{11}$ Antes de la inoculación se evaluó la viabilidad utilizando los colorantes vitales fluorogénicos: diacetato de fluoresceína (FDA), isotiocianato de fluoresceína (FITC) y yoduro de propidio (IP). ${ }^{7}$ Se observaron en un microscopio de epifluorescencia Olympus BH2. Una vez verificada su viabilidad, se diluyeron en solución salina fisiológica estéril, hasta obtener concentraciones de 0 , 6, 60, 600 y 6000 quistes. La concentración de 0 quistes se utilizó como control negativo.

Las ratas utilizadas fueron hembras y machos de la raza Sprague-Dawley, de 21 días de edad, recién destetadas, con pesos iniciales de 40 a 60 gramos; se 
colocaron en jaulas individuales de acero inoxidable; la temperatura del bioterio se mantuvo entre $25 \pm 2{ }^{\circ} \mathrm{C}$, con un rango de humedad relativa de 50 a $65 \%$ y un ciclo de luz oscuridad de 12 horas; se les ofreció agua $\mathrm{y}$ alimento ad libitum y se pesaron tres veces por semana durante el experimento. Antes de iniciar los experimentos se desinfectó el bioterio con luz ultravioleta, y las jaulas, charolas y comederos con cloro; las mamilas y el agua fueron esterilizadas en autoclave, durante 15 minutos a $121^{\circ} \mathrm{C}$.

Se realizó un diseño al azar para dos experimentos: a) Dieta de caseína a 10\% (DN ) y b) Dieta Libre de Nitrógeno (DLN) con cinco tratamientos en cada experimento, y cada uno tuvo las siguientes concentraciones: 0,6,60,600, y 6000 quistes/ml. Se utilizaron ocho animales experimentales para el primer experimento y seis para el segundo. Las unidades experimentales se asignaron al azar para cada tratamiento.

El tiempo de experimentación se estableció en 10 días de acuerdo con los informes existentes. ${ }^{4,5}$

En el caso de los animales con DLN, ésta se les suministró 10 días antes de la inoculación con el fin de provocar un estado de desnutrición. En ambos casos después de ser inoculadas se dieron alrededor de 10 a 11 días de experimentación.

El análisis estadístico de los datos obtenidos en relación con el peso y consumo de alimento se llevó a cabo utilizando el programa NCSS $6.0^{12}$ mediante la prueba de homogeneidad de varianza, análisis de varianza de un camino y análisis de residuales cuando fue necesario.

\section{Formulación de las dietas}

Se formularon dos dietas experimentales siguiendo las recomendaciones de la Association of Official Analytical Chemists (AOAC) para el Indice de Eficiencia Proteica (PER, por sus siglas en inglés). ${ }^{13}$ Dieta de caseína a $10 \%(\mathrm{DN})$ y dieta libre de nitrógeno (DLN). ${ }^{9}$ El propósito de la dieta DLN fue el de provocar una deficiencia proteica en las ratas y con esto una disminución en la respuesta inmunológica. El tipo de la dieta se muestra en el cuadro I. A cada una de las dietas preparadas se les analizó el contenido de humedad y de proteína, siguiendo los métodos descritos por el AOAC. ${ }^{14}$

\section{Inoculación}

Los animales fueron destetados a los 21 días de nacidos. A partir de ese momento, y durante tres días, se les ofreció una dieta (ad libitum) que cubría sus requerimientos. Al mismo tiempo se realizó un examen

\section{Cuadro I \\ Excreción de Quistes de Giardia lamblia de ratas ALIMENTADAS CON DIETAS DE CASEINA Y DIETA LIBRE DE nitrógeno, Hermosillo, Sonora, México, 1995-1999}

\begin{tabular}{|c|c|c|c|c|}
\hline \multirow[b]{2}{*}{$\begin{array}{c}\text { Concentración } \\
\text { de } \\
\text { inóculo }\end{array}$} & \multicolumn{4}{|c|}{ Porción de animales infectados } \\
\hline & $\begin{array}{c}\text { Caseína } \\
\text { (\# infectados/\# } \\
\text { total)* }\end{array}$ & $\begin{array}{c}\text { Porcentaje } \\
\text { de } \\
\text { infección }\end{array}$ & $\begin{array}{l}\text { Libre de nitrógeno } \\
\text { (infectados/\# } \\
\text { total)* }\end{array}$ & $\begin{array}{c}\text { Porcentaje } \\
\text { de } \\
\text { infección }\end{array}$ \\
\hline 0 & $0 / 8$ & $(0 \%)$ & $0 / 8$ & $(0 \%)$ \\
\hline 6 & $0 / 8$ & $(0 \%)$ & $0 / 8$ & $(0 \%)$ \\
\hline 60 & $1 / 8$ & (12.5\%) & $3 / 8$ & (37.5\%) \\
\hline 600 & $5 / 8$ & $(62.5 \%)$ & $6 / 8(75 \%)$ & $(37.5 \%)$ \\
\hline 6000 & $8 / 8$ & $(100 \%)$ & $8 / 8$ & $(100 \%)$ \\
\hline
\end{tabular}

* N úmero de ratas infectadas/ total de ratas en la concentración

coproparasitoscópico seriado de tres días. ${ }^{15}$ Las ratas elegidas para el ensayo fueron las que se encontraron libres de cualquier parásito y en las que se llevó a cabo la inoculación con los quistes de Giardia lamblia, los cuales fueron depositados en el estómago de la rata vía intraesofágica, utilizando agujas de alimentación previamente esterilizadas. El procedimiento se llevó a cabo en una campana de flujo laminar (Labconco Purifier Class II Safety Cabinet, modelo 36204-04). Al tercer día de la inoculación se inició el análisis coproparasitoscópico, día a día hasta terminar con el sacrificio de los animales.

\section{Evaluación histológica del intestino delgado de las ratas}

La presencia de quistes en las heces fue un indicador de la infección. Los animales infectados fueron sacrificados ${ }^{7,9}$ dos días después y se les practicó la disección para obtener el intestino delgado. El procedimiento fue realizado en una campana de flujo laminar y se utilizó material quirúrgico esterilizado.

El intestino delgado se dividió en tres partes: íleon (I), yeyuno (Y) y duodeno (D). La mitad de cada una de las tres porciones se utilizó para la observación de trofozoitos en el contenido intestinal, la otra mitad se usó para la observación histológica. Para la observación de trofozoitos se hicieron frotis con contenido intestinal y se tiñeron con Giemsa, para observarlos al microscopio y cuantificarlos.

La otra mitad se usó para la observación histológica, para lo cual las diferentes porciones del intestino se colocaron en una solución de glutaraldehído-formaldehído a 10\% (proporción 1:7 v/v) para fijarlos y observar los daños histológicos. 


\section{Resultados}

La incubación con yoduro de propidio no mostró quistes teñidos de rojo, por lo que se asumió que todos los quistes estaban viables.

La excreción de los quistes se inició a partir del $4^{\circ}$ día después de la inoculación, y una característica en las ratas de ambos experimentos (DN y DLN) fue la excreción intermitente de los quistes.

No se observó excreción de quistes, en el control negativo en los animales alimentados con dieta a 10\% de proteína (DN) e inoculados con seis quistes. Por otro lado, como se observa en el cuadro I, a medida que aumentó la concentración del inóculo, mayores fueron la excreción de quistes en las heces y el porcentaje de infección.

Por el contrario, en el grupo de animales alimentados con dieta DLN, se observó que el grupo inoculado con seis quistes presentó infección en $42.8 \%$ de los casos y se incrementó a medida que se aumentó la concentración de quistes (cuadro I).

En el grupo de dietas alimentadas con $\mathrm{DN}$ no se observaron cambios macroscópicos en ninguno de los grupos inoculados, incluyendo al grupo control. En los animales alimentados con DLN se observó que independientemente de la concentración del inóculo, presentaban una disminución significativa en el grosor de los intestinos, en comparación con los intestinos de las ratas alimentadas con DN.

En el grupo de animales alimentados con DN se observó la presencia de trofozoitos en las diferentes porciones del intestino delgado a partir de la concentración de 60 quistes, coincidiendo con lo observado en su excreción. En este grupo la sección del intestino que más se vio afectada fue el yeyuno, seguido por el íleon.

En los animales con dieta DLN la presencia de trofozoitos se detectó a partir de la concentración de seis quistes, observándose en las tres porciones del intestino delgado. Sin embargo, la porción intestinal en donde más proliferaron fue en el yeyuno, coincidiendo con lo encontrado en el grupo de animales con dieta $\mathrm{DN}$; y en menor proporción, proliferaron en el íleon.

La porción del duodeno no presentó daños microscópicos considerables en ninguno de los tratamientos del grupo DN. Los daños observados en el grosor de las vellosidades e indentaciones de las mismas se pueden observar en el cuadro II y en la figura 1. La porción del intestino más afectada en todos los casos fue el yeyuno, lo que coincide con la presencia de trofozoitos detectados en el contenido luminal. Las concentraciones de 600 y 6000 quistes fueron las que provocaron los daños más severos.

\section{Cuadro II \\ Daños observados en la mucosa INTESTINAL de RATAS QUE RECIBIERON UNA DIETA CON CASEINA A $10 \%$ Hermosillo, Sonora, México, 1995-1999}

\begin{tabular}{|c|c|c|c|c|c|c|}
\hline \multirow{3}{*}{$\begin{array}{l}\text { No. de quistes } \\
\text { inoculados }\end{array}$} & \multicolumn{6}{|c|}{ Porción del intestino delgado } \\
\hline & \multicolumn{2}{|c|}{ Duodeno } & \multicolumn{2}{|c|}{ Yeyuno } & \multicolumn{2}{|c|}{ lleon } \\
\hline & G & I & G & I & G & I \\
\hline 0 & + & - & - & - & $-1+$ & - \\
\hline 6 & - & $-1+$ & $-1+$ & $-1+$ & - & - \\
\hline 60 & - & $-1+$ & + & + & + & + \\
\hline 600 & + & + & + & + & $-(*)$ & + \\
\hline 6000 & - & $-1+$ & $++(*)$ & $-1+$ & $+t$ & ++ \\
\hline \multicolumn{3}{|c|}{$\begin{array}{l}\text { G = G rosor en las vellosidades } \\
(-)=\text { N egativo } \\
(+)=\text { Mínima intensidad } \\
(+++)=\text { Severa intensidad }\end{array}$} & \multicolumn{4}{|c|}{$\begin{array}{l}\text { I= Indentaciones en las vellosidades } \\
(-/+)=\text { Aislados } \\
(++)=\text { Moderada intensidad } \\
(*)=\text { Intenso edema subepitelial }\end{array}$} \\
\hline
\end{tabular}

Para el grupo LN los daños en la mucosa intestinal fueron muy severos en las tres porciones del intestino delgado (cuadro III y figura 2). En el grupo control (0 quistes) se observó un cierto daño en la mucosa del intestino con edema subepitelial. Por otro lado, la concentración de 60 quistes fue la que provocó un daño más severo a lo largo de todo el intestino delgado, observándose intenso edema subepitelial y cambios de gran intensidad en las microvellosidades, así como una excesiva cantidad de trofozoitos. Esto concuerda con el hallazgo de trofozoitos en el contenido intestinal.

El análisis de varianza efectuado mostró que la ganancia de peso entre los grupos alimentados con dieta $\mathrm{DN}$ fue estadísticamente diferente $(p<0.05)$. Sin embargo, al realizar una prueba de comparaciones múltiples (prueba de Duncan) los resultados obtenidos fueron contrarios a lo esperado, debido a que la ganancia de peso de los animales sometidos a concentraciones de 6 y 6000 tuvieron un comportamiento similar a los que no se inocularon. Por el contrario, las concentraciones de 60 y 600 les incrementaron su peso incluso más que en el grupo control; estos resultados tampoco coinciden con el estudio de presencia de trofozoitos, o porcentaje de infección, ya que fue en estas concentraciones en donde se observó su presencia.

El decremento de peso de los animales alimentados con DLN durante los 10 días previos al ensayo, cuando se realizó la inoculación, fue de $11.56 \pm 1.72 \mathrm{~g}$, provocándose una desnutrición en los animales con la intención de suprimir su estado inmunológico. El decremento de peso en los 10 días postinoculación fue de $8.8 \pm 1.32 \mathrm{~g}$, y el análisis estadístico efectuado 


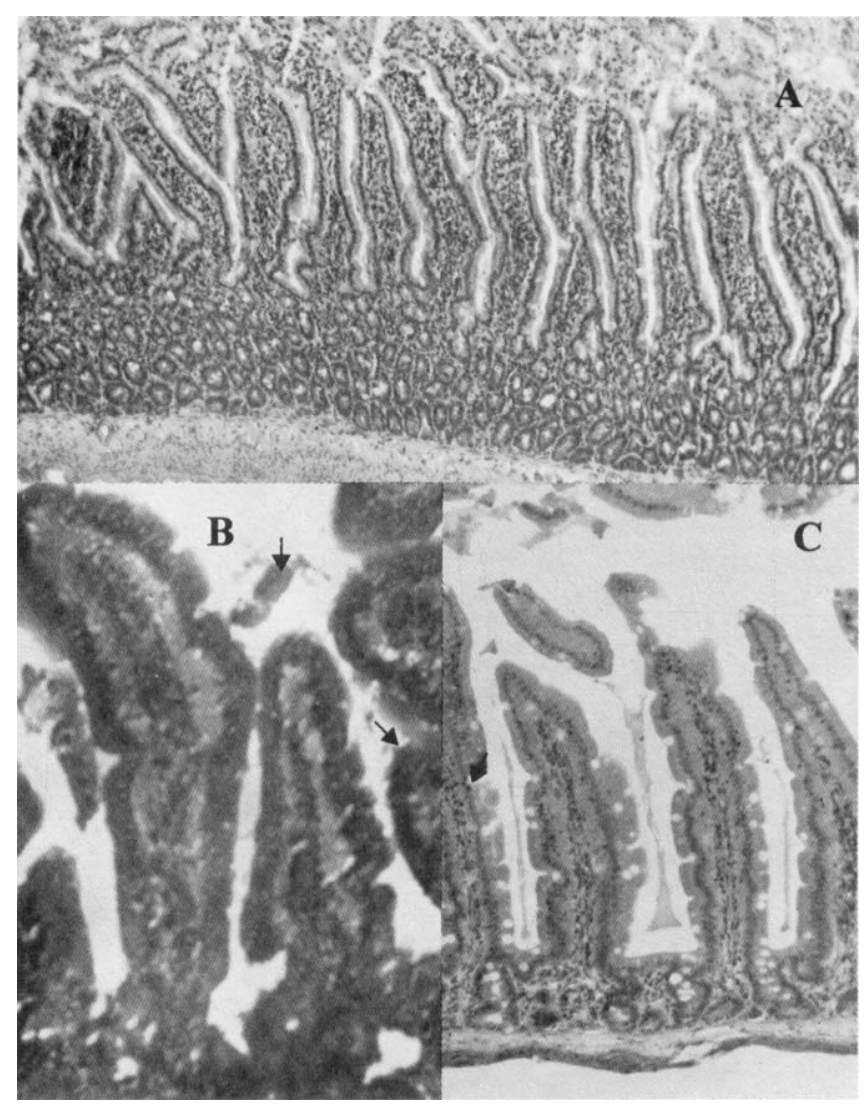

Figura 1. Cortes histológicos de intestinos de ratas. A) aspecto de un intestino de rata alimentada con DIETA DE CASEINA A $10 \%$ SIN INOCULAR (GRUPO CONTROL); SE OBSERVAN LAS CRIPTAS VELLOSAS COMPLETAS (40X); B) CORTE DE INTESTINO DE RATA ALIMENTADA CON DIETA DE CASEINA A 10\% INOCULADA CON 600 QUISTES; SE OBSERVAN ALGUNAS INDENTACIONES Y EL ACORTAMIENTO DE ALGUNAS CRIPTAS (100X). LAS FLECHAS INDICAN LA PRESENCIA DE TROFOZOITOS (100X);C) CORTE TRANSVERSAL DE YEYUNO DE RATA ALIMENTADA CON DIETA DE CASEÍNA A 10\%INOCULADA CON 6000 QUISTES SE OBSERVAN VELLOSIDADES DE TAMAÑo NORMAL PERO LA MAYORÍA CON INDENTACIONES

Cuadro III

Daños observados en la mucosa intestinal de RATAS QUE RECIBIERON UNA DIETA LIBRE DE NITRÓGENO, Hermosillo, Sonora, México, 1995-1999

\begin{tabular}{|c|c|c|c|c|c|c|}
\hline \multirow{3}{*}{$\begin{array}{l}\text { No. de quistes } \\
\text { inoculados }\end{array}$} & \multicolumn{6}{|c|}{ Porción del intestino delgado } \\
\hline & \multicolumn{2}{|c|}{ Duodeno } & \multicolumn{2}{|c|}{ Yeyuno } & \multicolumn{2}{|c|}{ IIleon } \\
\hline & G & I & G & I & G & I \\
\hline 0 & + & - & - & - & $-1+$ & - \\
\hline 0 & - & - & $-1+$ & $-1+$ & + & ++ \\
\hline 6 & $-(*)$ & t+ & $++(*)$ & ++ & t+ & - \\
\hline 60 & $+++\left({ }^{*}\right)$ & +++ & $+++\left({ }^{*}\right)$ & +++ & $+++(*)$ & +++ \\
\hline 600 & $-1+$ & + & $+t$ & + & $t+t$ & $-1+$ \\
\hline 6000 & - & ++ & $-1+$ & $-1+$ & + & - \\
\hline \multicolumn{3}{|c|}{$\begin{array}{l}G=\text { G rosor en las vellosidades } \\
(-)=N \text { egativo } \\
(+)=\text { Mínima intensidad } \\
(+++)=\text { Severa intensidad }\end{array}$} & \multicolumn{4}{|c|}{$\begin{array}{l}\text { I= Indentaciones en las vellosidades } \\
(-/+)=\text { Aislados } \\
(++)=\text { Moderada intensidad } \\
(*)=\text { Intenso edema subepitelial }\end{array}$} \\
\hline
\end{tabular}

en este punto para comparar los distintos tratamientos mostró una pequeña tendencia $(\mathrm{p}=0.07)$ a ser mayor el decremento a medida que aumentaba la concentración de quistes inoculados.

A pesar de encontrar diferencias significativas en la ganancia de peso de los animales sometidos a una dieta con caseína, los valores observados no concuerdan con los datos obtenidos en los daños a la mucosa, así como los de la proliferación de trofozoitos. En cuanto a los animales sometidos a una dieta libre de nitrógeno, la ganancia de peso no estuvo muy afectada; es posible que en esta etapa el organismo de la rata se encuentre ahorrando nutrimentos, lo cual hace difícil la detección de cambios de peso. En resumen, podemos decir que esta variable no parece ser un buen indicador de la presencia de infección. 


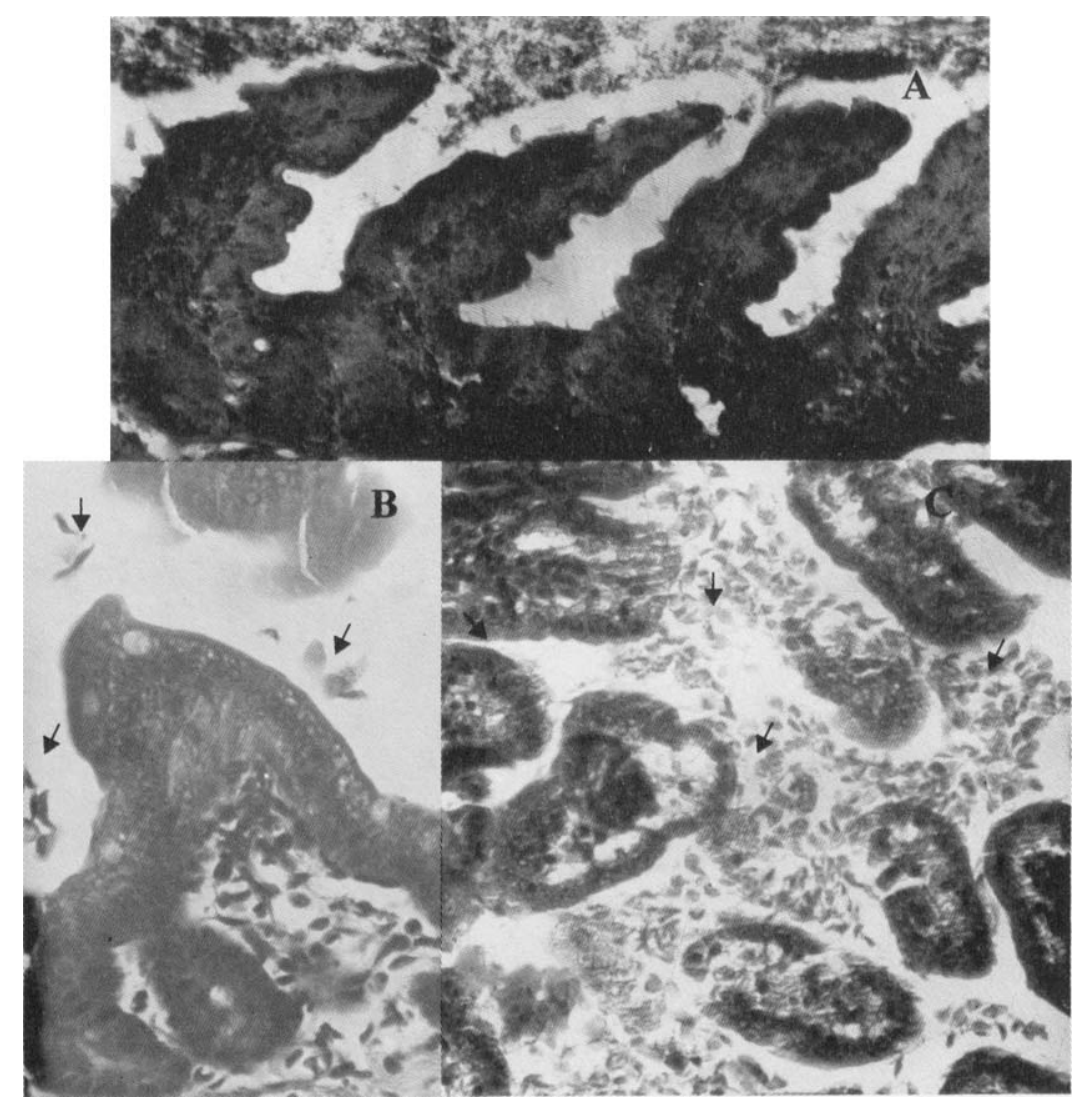

Figura 2. Cortes histológicos de intestinos de ratas. A) aspecto de un intestino de rata alimentada con Dieta DLN SIN INOCULAR (GRUPO CONTROL); SE OBSERVA CIERTO DAÑo EN LA MUCOSA DEL INTESTINO CON EDEMA SUBEPITELIAL; B) CORTE DE INTESTINO DE RATA ALIMENTADA CON DIETA LIBRE DE NITRÓGENO E INOCULADA CON 60 QUISTES; SE PUEDE OBSERVAR UNA CRIPTA COMPLETAMENTE ACHATADA Y DEFORME, ASIMISMO SE OBSERVAN LEUCOCITOS; C) CORTE TRANSVERSAL DE YEYUNO DE RATA ALIMENTADA CON DIETA LIBRE DE NITRÓGENO, SE PUEDEN OBSERVAR DATOS MAS SEVEROS. LAS FLECHAS INDICAN LA PRESENCIA DE TROFOZOITOS (100X)

\section{Discusión}

Este estudio revela que la carencia de proteína en la dieta de ratas Sprague-Dawley puede aumentar la susceptibilidad para la infección con dosis más pequeñas que las reportadas en otros modelos animales.

El grupo de animales alimentados con una dieta libre de nitrógeno necesitó únicamente de seis quistes para que se produjera la giardiosis. Esta situación pudo deberse a que la ausencia de proteína provocó una baja en el estado nutricional de los animales, lo que llevó a su vez a un deterioro del sistema inmunológico y los animales se volvieron más susceptibles a la infección. Esta deducción se basa en el hecho de que el estado nutricional no sólo tiene efectos apreciables sobre la inflamación y la respuesta a enfermedades infecciosas, sino que también tiene una función crucial sobre los mecanismos de homeostasis controlados por el sistema. ${ }^{16,17}$ La malnutrición proteico-enérgetica está asociada con un daño significativo de la inmunidad mediada por células, función fagocítica, sistema complemento, concentración de anticuerpos secretores de inmunoglobulina A y producción de citocinas. ${ }^{18} \mathrm{De}$ igual manera, esta condición dietaria ha mostrado en estudios in vitro que la proliferación celular y la síntesis de $\mathrm{ADN}$, se reducen. ${ }^{19}$

En cuanto a la excreción, ésta se inició al cuarto día concordando con los resultados obtenidos por Craft, ${ }^{9}$ y Belosevic y colaboradores, ${ }^{8}$ quienes inocularon jerbos y observaron la excreción al octavo día. Por 
su parte, Schupp y colaboradores, ${ }^{7}$ trabajando con ratones neonatos, detectaron la presencia de quistes en las heces al quinto día.

En el presente trabajo la excreción de quistes se presentó de manera intermitente. Estos resultados coinciden con los obtenidos por Belosevic y colaboradores, ${ }^{8}$ quienes trabajando con jerbos también observaron intermitencia en esta especie. Empero, Roberts Thompson y colaboradores, ${ }^{7}$ reportaron que ratones inoculados con Giardia muris no presentaban intermitencia.

De igual manera, en este estudio la porción del intestino delgado que más se vio afectada por la presencia de trofozoitos fue el yeyuno, seguido por el íleon. Sin embargo, Campbell y colaboradores, no observaron una distribución uniforme de trofozoitos en jerbos infectados.

Es importante señalar la relevancia de la dieta en la dosis infectiva, ya que en la DLN se logró infectar a los animales con sólo seis quistes $/ \mathrm{ml}$. En humanos se ha reportado una dosis infectiva de 10 quistes.

El impacto y relevancia aumenta cuando se consideran los múltiples reportes de agua contaminada con Giardia lamblia ${ }^{20-23}$ y el número de niños y otras personas desnutridas en países en desarrollo, ${ }^{3}$ y la interferencia que tiene este parásito con la absorción de algunos nutrimentos. Además, si transferimos estos resultados al ser humano, el yeyuno es la sección en donde se absorben nutrimentos como glucosa, galactosa, fructosa, vitamina $\mathrm{C}$, tiamina, riboflavina, piridoxina y ácido fólico. Aunado a esto, uno de los síntomas más comunes de la giardiosis en el humano es la mala absorción de azúcares, los cuales son fermentados por bacterias causando flatulencia, distensión intestinal y dolor. En el Ileon se absorben proteínas, vitaminas liposolubles, grasas y colesterol, lo que causa mala absorción de grasas y esto provoca esteatorrea, muy común en la giardiosis. Las observaciones macro y microscópicas del intestino delgado del grupo de animales con DLN indican una susceptibilidad mayor a la infección con giardia y que muy probablemente se deba a la falta de proteína en la dieta. ${ }^{18}$ De acuerdo con Watson, las alteraciones nutricias ocasionan daños o alteraciones celulares severas en las funciones humorales..$^{23}$

Con base en los resultados se obtienen las siguientes conclusiones:

1. La excreción de quistes se inició al cuarto día, de manera intermitente, y fue independiente del tipo de dieta

2. El porcentaje de infección dependió de la concentración del inóculo
3. La dosis infectiva fue dependiente de la dieta; con animales desnutridos se reduce hasta seis quistes

4. Las secciones del intestino más dañadas fueron yeyuno e íleon, y esto se evidenció más en animales desnutridos en la sección del duodeno, y

5. La medición de la ganancia de peso a 10 días no resultó un buen indicador del grado de infección por Giardia lamblia.

\section{Agradecimientos}

Se agradece la colaboración del Dr. Enrique RamosSalas, por el apoyo estadístico y al médico patólogo Guillermo López Cervantes, por su apoyo en la evaluación de los cortes histológicos.

\section{Referencias}

1.W olfe MS. Giardiasis. C lin Microbiol Rev 1992;5(1):93-100.

2. 0 verturf GD. Endemic giardiasis in the United States. Role of the day care center. C lin Infect D is 1994;18:764-765.

3.Valencia ME, Jardínes RP, N oriega GE,W ong P, G rijalva MI, C ruz MR et al. Estudio de la zona serrana del estado de Sonora. Hermosillo, Sonora: Instituto de Investigaciones y Estudios Superiores de N oroeste, 1980; Reporte Técnico 80-001.

4.Valencia ME, Jardines RP, N oriega ED GE, W ong P, Grijalva MI, Cruz MR et al. Estado nutricional en los centros urbanos marginados de Sonora. Hermosillo, Sonora: Instituto de Investigaciones y Estudios Superiores de Noroeste, 1980; 1981 Reporte Técnico 81-002.

5. Díaz-C inco ME,A guilar-G arcía JM, Fraga RE,A cedo FE. Incidence of Giardia lamblia in finished potable water. Proceedings of the $84^{\text {th }} A$ nnual Meeting of the International Association of Milk Food and Environmental Sanitarians; 1997 Jul, 0 rlando (FL), U nited States of America.

6. Astiazarán $\mathrm{H}$. Evaluación bioquímica-nutricional de vitamina $\mathrm{A}$ y hierro en niños con giardiasis asistiendo a la consulta externa del Hospital Infantil del Estado de Sonora (tesis). Hermosillo, Sonora: Universidad de Sonora, 1988.

7. Roberts-Thomson IC, Stevens D P, Mahmoud AA, W arren KS. G iardiasis in the mouse:An animal model. Gastroenterology 1976;71(1):57-61.

8. Belosevic M, Faubert G M, MacLean JD, Law C, C roll N A. Giardia lamblia infections in Mongolian gerbils: An animal model. J Infect Dis 1983; 147(2):222-226.

9. Craft CJ. Experimental infection with Giardia lamblia in rats.J infect $D$ is 1982;145(4):495-498.

10. Makled MKHMI, Manar MSA, Salwaf AES, A mira KI. Effect of immunosuppression of the virulence of Giardia lamblia cysts.J Egyptian Soc Parasitol 1994;24(1):205-210.

11. Bernard HJ, ed. Diagnóstico y tratamiento clínicos por el laboratorio. Tomo II. 8a ed. México, D.F.: Salvat Editores, 1990.

12. Hintze JL. Q uick start and self help manual. N C SS 97 statystical system for W indows, 1997.

13. 0 fficial Methods of Analysis of the Association of 0 fficial Analytical Chemists. 14 ${ }^{\text {th }}$ edition. Arlington (VA):AO AC 1984:22-209.

14. Bender AE, D oell BM. Biological evaluation of proteins:A new aspect. $B$ J N utr 1957;11:140-148.

15.Vázquez TO,Velazco C 0. Giardiasis. Infectol 1987;7(4):169-178. 
16. Sánchez-Alvarez V. Inmunocompetencia en la malnutrición proteicoenergética. Rev Cubana Aliment N utr 1999;13(2):129-136.

17. D eplancke B, G skins H R. Microbial modulation of innate defense: $G$ oglet cells and intestinal mucus layer. Am J C lin N utr 2001;73:1131S-1141S.

18. Chandra-Ranjit K. N utrition and Immune system:An introduction.Am I Clin N utr 1997;66:460S-463S.

19. Sherman AR. Zinc, cooper and iron nutriture and immunity. J Nutr 1992;122:604-609.

20. Campbell JD, Faubert GM. Comparative studies on Giardia lamblia encystation in vitro and in vivo.J Parasitol 1994;80(1):36-44.
21. A kin EW, Jakubowski W. D rinking water transmission of giardiasis in the United States.W ater Sci Technol 1986;18(10):219-226.

22. Shaw PK, Brodsky RE, Lyman D O,W ood BT, Hibler CP, Healy GR et al. A communitywide outbreak of giardiasis with evidence of transmission by a municipal water supply. Ann Intern Med 1977;87(4):426-432.

23.W atson RR. Resistance to intestinal parasites during murineA ID S: Role of alcohol and nutrition in immune dysfunction. Parasitology 1993;107 (Suppl):S69-S74. 